MATHEMATICS OF COMPUTATION

Volume 75, Number 256, October 2006, Pages 1871-1889

S $0025-5718(06) 01867-9$

Article electronically published on June 22, 2006

\title{
WAVELETS WITH PATCHWISE CANCELLATION PROPERTIES
}

\author{
HELMUT HARBRECHT AND ROB STEVENSON
}

\begin{abstract}
We construct wavelets on general $n$-dimensional domains or manifolds via a domain decomposition technique, resulting in so-called composite wavelets. With this construction, wavelets with supports that extend to more than one patch are only continuous over the patch interfaces. Normally, this limited smoothness restricts the possibility for matrix compression, and with that the application of these wavelets in (adaptive) methods for solving operator equations. By modifying the scaling functions on the interval, and with that on the $n$-cube that serves as parameter domain, we obtain composite wavelets that have patchwise cancellation properties of any required order, meaning that the restriction of any wavelet to each patch is again a wavelet. This is also true when the wavelets are required to satisfy zeroth order homogeneous Dirichlet boundary conditions on (part of) the boundary. As a result, compression estimates now depend only on the patchwise smoothness of the wavelets that one may choose. Also taking stability into account, our composite wavelets have all the properties for the application to the (adaptive) solution of well-posed operator equations of orders $2 t$ for $t \in\left(-\frac{1}{2}, \frac{3}{2}\right)$.
\end{abstract}

\section{Motivation AND BACKGROUnd}

For some $n^{\prime} \geq n \geq 1$, let $\Omega$ be an $n$-dimensional manifold in $\mathbb{R}^{n^{\prime}}$. We are interested in approximating the solution of an equation $L u=f$, where for some Hilbert space $H$ of functions on $\Omega$, typically being a Sobolev space, with dual $H^{\prime}$, $L: H \rightarrow H^{\prime}$ is boundedly invertible, and $f \in H^{\prime}$. When $\Omega$ is a domain in $\mathbb{R}^{n}$, we think of the equation as being the result of a variational formulation of a boundary value problem, and when it is a true manifold, we have in mind an integral equation formulated on the boundary of an $(n+1)$-dimensional domain.

Now let us assume that we have available a Riesz basis $\Psi$ for $H$ of wavelet type, where each wavelet is assumed to have the cancellation property of a certain order, meaning that, possibly after making some smooth transformation of coordinates, it is orthogonal to all polynomials of that order.

Thinking of strongly elliptic problems, for any $V \subset H$ spanned by some finite subset of the wavelets, we can approximate $u$ by the Galerkin solution from $V$. This approach has two attractive features. First, since $\Psi$ is a Riesz basis, the stiffness matrix with respect to the wavelet basis is well conditioned uniformly in $V$, allowing an efficient iterative solution. Second, for $L$ being a singular integral

Received by the editor October 8, 2004 and, in revised form, March 14, 2005.

2000 Mathematics Subject Classification. Prmary 46B15, 46E35, 65N55, 65T60.

Key words and phrases. Wavelets, domain decomposition, patchwise cancellation properties, matrix compression, Riesz bases.

This work was supported by the Netherlands Organization for Scientific Research and by the EC-IHP project "Breaking Complexity".

(c)2006 American Mathematical Society Reverts to public domain 28 years from publication 
operator, the cancellation property of a sufficiently high order of the wavelets allows us to compress the stiffness matrix, which in this case is dense, to a sparse one without qualitatively affecting the discretization error. The compression, i.e., the dropping of certain entries, applies, in different forms, to two types of entries. The so-called first compression applies to pairs of wavelets that have supports with a sufficiently large mutual distance, whereas, thinking of piecewise smooth wavelets, the second compression applies to pairs of wavelets that have overlapping supports, but for which the support of one wavelet living on the higher level is sufficiently far away from the singular support of the other one living on a lower level (see [Sch98, DHS02]). Together, the well conditionedness of the stiffness matrix and, for singular integral operators, the compression of this matrix allow us to find an approximate solution of the Galerkin system in $\mathcal{O}(\# V)$ operations with an error that, up to some absolute constant factor, is as good as that of the exact solution of this system.

Instead of computing Galerkin approximations from fixed, finite-dimensional subspaces $V$, as exposed in CDD01, CDD02, the availability of a wavelet basis $\Psi$ opens a way to approximate the solution $u$ using an adaptive scheme. Since $\Psi$ is a Riesz basis for $H$, the equation $L u=f$ has an equivalent formulation as an infinite, well-posed (in $\ell_{2}$-metric) matrix-vector system, which is formally the Galerkin system with " $V$ " equal to $H$, equipped with the wavelet basis. Now, coarsely speaking, the idea is to apply a simple iterative scheme, like Richardson iteration, directly to this infinite system, where in each iteration the application of the matrix is replaced by that of an adaptively compressed matrix. Apart from the second compression, and for integral operators, the first compression, it is now necessary to consider a third compression that applies to pairs of wavelets for which the support of the wavelet on the higher level intersects the singular support of the wavelet on the lower level. Indeed, note that, even for differential operators, without either the second or third compression one is left with infinitely many nonzero entries in each column. The decay of the entries, as meant in the third compression, as a function of the difference in levels of the wavelets involved, relies not only on the cancellation property of the wavelet on the higher level, but also on a sufficient global smoothness of the wavelet on the lower level. Essentially only for spline wavelets, which have maximal smoothness in relation to their approximation orders, the compression error can be shown to be sufficiently small so that the adaptive wavelet method has optimal computational complexity, in the sense that the approximations yielded by this algorithm converge with the same rate as that of socalled best $N$-term approximations, taking only a number of arithmetic operations that is proportional to the vector length (see [Ste04b], and, for the computation of the required entries using quadrature, see [GS04, GS05]).

In view of the above applications, it is by now well known how to construct wavelets on the line ([CDF92]) and with additional efforts on the interval ([DKU99]), and so using tensor products on $n$-cubes that, properly scaled, generate Riesz bases for a range of Sobolev spaces, have the cancellation property of any required order, and are sufficiently smooth in relation to their approximation order. The challenge is to construct such wavelets on more general domains or manifolds $\Omega$. The most well-known method is via a domain decomposition approach (see DS99a, and CTU99, CM00] for related techniques). With this approach, $\Omega$ is written as $\bar{\Omega}=\bigcup_{q=1}^{M} \bar{\Omega}_{q}$, where $\Omega_{q} \cap \Omega_{q^{\prime}}=\emptyset$ for $q \neq q^{\prime}, \Omega_{q}=\kappa_{q}(\square)$, where the 
$\kappa_{q}: \mathbb{R}^{n} \rightarrow \mathbb{R}^{n^{\prime}}$ are smooth, regular parametrizations, and $\square=(0,1)^{n}$. Primal and dual scaling functions constructed on $\square$ are lifted to the $\Omega_{q}$, and are continuously connected over the interfaces. With respect to the modified $L_{2}(\Omega)$-scalar product $\langle\langle u, v\rangle\rangle=\sum_{q} \int_{\square}\left(u \circ \kappa_{q}\right)(x)\left(v \circ \kappa_{q}\right)(x) d x$, the resulting global primal and dual scaling functions are biorthogonal. The "composite" wavelets are now obtained by subtracting the biorthogonal projection from collections of functions spanning "initial" complement spaces between any two successive spaces in the primal multiresolution analysis. These collections are simply obtained by lifting such collections on $\square$. Although this construction realizes wavelets on general domains or manifolds that, properly scaled, generate Riesz bases for a range of Sobolev spaces, it also has some limitations:

- wavelets with supports that extend to more than one patch generally have no cancellation property with respect to the canonical $L_{2}$-scalar product,

- the wavelets generate Riesz bases for Sobolev spaces $H^{s}$ generally only for $s>-\frac{1}{2}$ (and $s<\frac{3}{2}$ ),

- wavelets with supports that extend to more than one patch are only continuous,

where the first two limitations are a consequence of the fact that biorthogonality is realized with respect to the modified $L_{2}$-scalar product.

These limitations were already recognized in DS99a, and in DS99b the same authors developed an elegant approach to construct wavelets on general domains or manifolds that, properly scaled, generate Riesz bases for $H^{s}$ for in principal any $s$, if not restricted by the regularity of the manifold, and that have the cancellation property of any desired order. Unfortunately, so far with this approach it does not seem easy to construct wavelets that have competitive quantitative properties. However, new results in this direction have been recently reported in KS04.

By an adaptation of the construction from DS99a, in Ste04a] we constructed composite wavelets that generate Riesz bases for the Sobolev spaces $H^{s}$ for the full range of $s$ that is allowed by the continuous gluing of functions over the patch interfaces (i.e., $|s|<\frac{3}{2}$ ), if not restricted by the regularity of the manifold, and that all have the cancellation property of any desired order. These wavelets have all properties required for the earlier mentioned application of solving Galerkin systems resulting from differential or singular integral operators with orders $2 t$ when $|t|<\frac{3}{2}$. For the application in the adaptive wavelet method, however, the limited smoothness of wavelets over patch interfaces has an adverse affect on the third compression, and optimality of that scheme can only be shown for wavelets with relatively small approximations orders $d<\frac{3 n / 2-t}{n-1}$ (Ste04b, Remark 2.4]).

In the present paper, we construct composite wavelets that have the stronger patchwise cancellation property of any desired order, meaning that the restriction of any wavelet to any patch $\Omega_{q}$ has the cancellation property of that order. This property will also hold when, in case the manifold has a boundary, at the primal side a zeroth order homogeneous Dirichlet boundary condition is prescribed on (part of) this boundary. We will enforce the patchwise cancellation property, say of order $\tilde{d}$, by modifying the dual scaling functions on the interval $(0,1)$ such that already the interior ones, i.e., those that will not be glued over patch interfaces, span the full space $P_{\tilde{d}-1}(0,1)$. Obviously, the patchwise cancellation property implies the cancellation property with respect to the canonical $L_{2}$-scalar product. What is more, although also with this construction wavelets with supports that intersect 
patch interfaces are only continuous, in any case for differential operators, thanks to the patchwise cancellation property, this limited smoothness does not affect the third compression. Indeed, for such an operator, say of order $2 t$ with $t<\frac{3}{2}$, an entry in the infinite stiffness matrix corresponding to wavelets $\psi_{\lambda}, \psi_{\mu}$ is of the form $\int_{\Omega} \sum_{|\alpha|,|\beta| \leq t} a_{\alpha, \beta} \partial^{\alpha} \psi_{\lambda} \partial^{\beta} \psi_{\mu}=\sum_{q} \int_{\Omega_{q}} \sum_{|\alpha|,|\beta| \leq t} a_{\alpha, \beta} \partial^{\alpha} \psi_{\lambda} \partial^{\beta} \psi_{\mu}$. Now, in principal for any order of the wavelets, the above right-hand side shows that with only a sufficient patchwise smoothness, as with (lifted) splines, and with a patchwise cancellation property of a sufficiently high order, the appropriate decay of the entry is ensured as a function of the difference $\| \lambda|-| \mu||$ in levels and, with that, the optimality of the adaptive wavelet scheme.

The patchwise cancellation property also induces a limitation. Thinking of a domain $\Omega$ being simply the union of $n$-cubes $\Omega_{q}$, this property means that any wavelet $\psi_{\lambda}$, as always except those with level $|\lambda|=0$ which are scaling functions, restricted to each $\Omega_{q}$ is orthogonal to all polynomials of degree $\tilde{d}-1$. Assuming, as will be ensured, that the collection $\Psi=\left\{\psi_{\lambda}: \lambda \in \Lambda\right\}$ is a Riesz basis for $L_{2}(\Omega)$, with dual basis $\tilde{\Psi}$, then for any $v \in \prod_{q} P_{\tilde{d}-1}\left(\Omega_{q}\right) \subset L_{2}(\Omega)$ we have that $v=\sum_{\lambda \in \Lambda}\left\langle v, \psi_{\lambda}\right\rangle \tilde{\psi}_{\lambda}=\sum_{\lambda \in \Lambda,|\lambda|=0}\left\langle v, \psi_{\lambda}\right\rangle \tilde{\psi}_{\lambda}$, so that apparently $\prod_{q} P_{\tilde{d}-1}\left(\Omega_{q}\right)$ is in the span of the dual scaling functions on the lowest level. So necessarily, all dual spaces contain functions that have jumps across interfaces between patches, from which we infer, regardless of whether biorthogonality is realized with respect to the canonical or the modified $L_{2}$-scalar product, that the (primal) wavelets cannot generate Riesz bases for $H^{s}$ when $s \leq-\frac{1}{2}$. We will ensure, however, that, properly scaled, our wavelets generated Riesz bases for $H^{s}$ for $s \in\left(-\frac{1}{2}, \frac{3}{2}\right)$, which, apart from the application in the adaptive wavelet scheme, makes them suitable for the solution of Galerkin systems resulting from differential or singular integral operators of orders $2 t$, with $t \in\left(-\frac{1}{2}, \frac{3}{2}\right)$.

This paper is organized as follows. In Section 2, we collect the assumptions on the primal and dual multiresolution analyses on the interval. We propose two constructions of modified scaling functions that will both eventually lead to composite wavelets with patchwise cancellation properties. In Section 3 we construct multiresolution analyses on the $n$-cube simply by means of tensor products. Following the approach from [DS99a], in Section 4 we construct composite wavelets on general domains or manifolds that, thanks to the modification of the scaling functions, have patchwise cancellation properties. Finally, in Section 5, we illustrate our approach by constructing on a simple L-shaped domain, wavelets of order 2 that have patchwise cancellation properties of order 2 .

In order to avoid the repeated use of generic but unspecified constants, in this paper by $C \lesssim D$ we mean that $C$ can be bounded by a multiple of $D$, independent of parameters on which $C$ and $D$ may depend. Obviously, $C \gtrsim D$ is defined as $D \lesssim C$, and $C \approx D$ as $C \lesssim D$ and $C \gtrsim D$.

For $H$ being a separable Hilbert space with scalar product $\langle\cdot, \cdot\rangle$ and norm $\|\cdot\|$, and for a countable collection $\Sigma$ of functions in $H$, which we formally view as a (column) vector, and for $\mathbf{c}=\left[c_{\sigma}\right]_{\sigma \in \Sigma}$ a vector of scalars, by $\mathbf{c}^{T} \Sigma$ we will mean the expansion $\sum_{\sigma \in \Sigma} c_{\sigma} \sigma$. The span of $\Sigma$ will be denoted as $\mathcal{S}(\Sigma)$. For $x \in H$, with $\langle\Sigma, x\rangle$ and $\langle x, \Sigma\rangle$ we will mean the column- and row-vectors with coefficients $\langle\sigma, x\rangle$ and $\langle x, \sigma\rangle, \sigma \in \Sigma$. When $\tilde{\Sigma}$ is another countable collection in $H$, with $\langle\Sigma, \tilde{\Sigma}\rangle$ we denote the matrix $(\langle\sigma, \tilde{\sigma}\rangle)_{\sigma \in \Sigma, \tilde{\sigma} \in \tilde{\Sigma}}$. For $V \subset H$ being a dense, continuously embedded Banach space, as usual we will sometimes also use $\langle\cdot, \cdot\rangle$ to denote the 
duality pairing $\langle\cdot, \cdot\rangle_{V \times V^{\prime}}$, which, with the aforementioned meaning, can also be applied to collections from $V$ and/or $V^{\prime}$.

On the spaces of (possibly infinite) scalar vectors or matrices, we will exclusively use the $\ell_{2}$-scalar product, $\ell_{2}$-norm, or the resulting operator norm, that we therefore simply denote by $\langle\cdot, \cdot\rangle$ or $\|\cdot\|$, respectively. A collection $\Sigma$ is called a Riesz system when $\left\|\mathbf{c}^{T} \Sigma\right\| \bar{\sim}\|\mathbf{c}\|$, i.e., when $\langle\Sigma, \Sigma\rangle$ is boundedly invertible, and $\Sigma$ is called a Riesz basis when it is in addition a basis for $H$. When $\Sigma$ depends on a parameter, we will speak about uniform Riesz systems (or bases) when the above equivalence holds uniformly over the values this parameter may attain.

\section{Biorthogonal multiResolution on $(0,1)$}

2.1. Assumptions. Throughout this section, let $\langle\cdot, \cdot\rangle=\langle\cdot, \cdot\rangle_{L_{2}(0,1)}$ and $\|\cdot\|=$ $\|\cdot\|_{L_{2}(0,1)}$. For some fixed $m \in \mathbb{Z}$ and all $j \in \mathbb{Z}_{m}:=\mathbb{Z} \cap[m, \infty)$, we assume an index set $\{0,1\} \subset I_{j} \subset[0,1]$ with $x \in I_{j}$ if and only if $1-x \in I_{j}$, and $\sup _{k \in \mathbb{N}_{0}} \#\left(I_{j} \cap\left[k 2^{-j},(k+1) 2^{-j}\right]\right) \lesssim 1$. For $j \in \mathbb{Z}_{m}$, we assume collections of uniformly $L_{2}(0,1)$-bounded primal and dual scaling functions $\Phi_{j}=\left[\phi_{j, x}\right]_{x \in I_{j}}$ and $\tilde{\Phi}_{j}=\left[\tilde{\phi}_{j, x}\right]_{x \in I_{j}}$ that satisfy

$$
\begin{aligned}
& \phi_{j, x}(y)=0 \text { if not }|x-y| \lesssim 2^{-j}, \\
& \phi_{j, x}(\cdot)=\phi_{j, 1-x}(1-\cdot),
\end{aligned}
$$

$(\mathcal{J}) \quad$ for some $d \in \mathbb{N}, P_{d-1}(0,1) \subset \mathcal{S}\left(\Phi_{j}\right)$,

$(\mathcal{N}) \quad \mathcal{S}\left(\Phi_{j}\right) \subset \mathcal{S}\left(\Phi_{j+1}\right)$

$(\mathcal{B}) \quad$ for some $\gamma>0$, and any $s \in[0, \gamma)$,

$$
\|\cdot\|_{H^{s}(0,1)} \lesssim 2^{s j}\|\cdot\| \quad \text { on } \mathcal{S}\left(\Phi_{j}\right),
$$

with analogous conditions on $\left(\tilde{\Phi}_{j}\right)_{j}$, denoted as $(\tilde{\mathcal{L}}),(\tilde{\mathcal{S}}),(\tilde{\mathcal{J}}),(\tilde{\mathcal{N}})$, and $(\tilde{\mathcal{B}})$, with $(d, \gamma)$ replaced by generally different parameters $(\tilde{d}, \tilde{\gamma})$. We assume that the primal scaling functions are continuous and satisfy

$$
\phi_{j, x}(0)=0 \text { for } x \in I_{j} \backslash\{0\} .
$$

Finally, we assume that the primal and dual scaling functions are biorthogonal, i.e., that

$$
\left\langle\Phi_{j}, \tilde{\Phi}_{j}\right\rangle=I
$$

Although in applications it is important to select the minimal level $m$ as small as possible (see BF01] for an approach), for convenience, in order to be not forced to handle exceptional cases corresponding to the coarsest levels, we will assume that $m$ is sufficiently large .

From the assumptions on $I_{j}$, the uniform boundedness of all scaling functions, $(\underline{\mathcal{L}}),(\tilde{\mathcal{L}})$, and $(\mathcal{D})$, it follows that both $\Phi_{j}$ and $\tilde{\Phi}_{j}$ are uniform $L_{2}(0,1)$-Riesz systems (see, e.g., DKU99, Lemma 2.1]).

In addition, for $j \in \mathbb{Z}_{m+1}$, we assume an index set $J_{j} \subset(0,1)$ with $J_{j} \cap I_{j}=\emptyset$, $x \in J_{j}$ if and only if $1-x \in J_{j}$, and $\sup _{k \in \mathbb{N}_{0}} \#\left(J_{j} \cap\left[k 2^{-j},(k+1) 2^{-j}\right]\right) \lesssim 1$. For $j \in \mathbb{Z}_{m+1}$, we assume collections

$$
\Xi_{j}=\left[\xi_{j, x}\right]_{x \in J_{j}} \subset \mathcal{S}\left(\Phi_{j+1}\right)
$$


that satisfy corresponding conditions $(\underline{\mathcal{L}}),(\underline{\mathcal{S}})$, and $(\mathcal{V})$, the latter meaning that all $\xi_{j, x}$ vanish at $\{0,1\}$, such that

$$
\Upsilon_{j+1}:=\left[\begin{array}{ll}
\Phi_{j}^{T} & \Xi_{j}^{T}
\end{array}\right]^{T} \text { is a uniform } L_{2}(0,1) \text {-Riesz basis for } \mathcal{S}\left(\Phi_{j+1}\right)
$$

and

$$
\left\langle\Upsilon_{j+1}, \tilde{\Phi}_{j+1}\right\rangle^{-1} \text { is uniformly local, }
$$

by which we mean that only entries of this matrix indexed by $(x, y)$ with $|x-y| \lesssim$ $2^{-j}$ might be nonzero. The last condition is only needed if one is also interested in having locally supported dual wavelets. By $\Upsilon_{j+1}=\left\langle\Upsilon_{j+1}, \tilde{\Phi}_{j+1}\right\rangle \Phi_{j+1}$, note that $\left\langle\Upsilon_{j+1}, \tilde{\Phi}_{j+1}\right\rangle^{-T}$ represents the transformation from $\Phi_{j+1}$ to the two-level basis $\Upsilon_{j+1}$.

Remark 2.1. In DS99a], the matrices $\left\langle\Upsilon_{j+1}, \tilde{\Phi}_{j+1}\right\rangle^{T}$ and $\left\langle\Upsilon_{j+1}, \tilde{\Phi}_{j+1}\right\rangle^{-T}$ are denoted as $\breve{\mathbf{M}}_{j}$ and $\breve{\mathbf{G}}_{j}$, respectively. The completion of $\Phi_{j}$ by $\Xi_{j}$ to a uniform $L_{2}$-Riesz basis for $\mathcal{S}\left(\Phi_{j+1}\right)$, or equivalently, the completion of the $\# I_{j+1} \times \# I_{j}$ matrix $\left\langle\Phi_{j}, \tilde{\Phi}_{j+1}\right\rangle^{T}$ by the $\# I_{j+1} \times \# J_{j}$ matrix $\left\langle\Xi_{j}, \tilde{\Phi}_{j+1}\right\rangle^{T}$ to a uniformly wellconditioned matrix $\left\langle\Upsilon_{j+1}, \tilde{\Phi}_{j+1}\right\rangle^{T}$, is known as an (initial) stable completion (see [CDP96]).

For any $2 \leq d \leq \tilde{d}$ with $d+\tilde{d}$ even and $\tilde{d}$ sufficiently large, collections $\Phi_{j}, \tilde{\Phi}_{j}$ that satisfy all above assumptions with $\gamma=d-\frac{1}{2}$ and $\tilde{\gamma}$ growing proportionally with $\tilde{d}$ were constructed in DKU99]. For some parameter $\mathbb{N} \ni r \geq d-1+d \bmod 2$ that one may choose, the primal collections $\Phi_{j}$ span the standard spline space of order $d$ with respect to the knot sequence

$$
(\underbrace{0, \ldots, 0}_{d \text { times }}, r 2^{-j}, r 2^{-j}+2^{-j}, \ldots, 1-r 2^{-j}, \underbrace{1, \ldots, 1}_{d \text { times }}) .
$$

As collections $\Xi_{j}$ one may take bases for the biorthogonal complement spaces $\mathcal{S}\left(\Phi_{j+1}\right) \cap \mathcal{S}\left(\Phi_{j}\right)^{\perp_{L_{2}(0,1)}}$, i.e., collections of biorthogonal wavelets on the interval as constructed in [DKU99], which, in view of (ㄱ) , have to be modified by subtracting from those wavelets that do not vanish at 0 or 1 a suitable multiple of $\phi_{j-1,0}$ or $\phi_{j-1,1}$ (cf. DS99a, Remark 2.4.2]). These biorthogonal wavelets themselves were constructed by subtracting biorthogonal projections from simpler initial stable completions $\Xi_{j}$. Alternatively, just these completions can be applied, which, for $n>1$, actually lead to composite wavelets with smaller supports (cf. [Har01, HS04]).

2.2. Modified scaling functions. We are going to construct sequences of collections of modified primal and dual scaling functions $\left(\Phi_{j}^{\text {new }}\right)_{j \in \mathbb{Z}_{m}}$ and $\left(\tilde{\Phi}_{j}^{\text {new }}\right)_{j \in \mathbb{Z}_{m}}$ that span the original spaces, satisfy $(\mathcal{D})$, as well as all of $(\mathcal{L})-(\mathcal{V})$ or $(\tilde{\mathcal{L}})-(\tilde{\mathcal{B}})$, respectively, and, in addition, for which

$$
P_{\tilde{d}-1}(0,1) \subset \mathcal{S}\left(\tilde{\Phi}_{j}^{\text {new }} \backslash\left\{\tilde{\phi}_{j, 0}^{\text {new }}, \tilde{\phi}_{j, 1}^{\text {new }}\right\}\right),
$$

and, with $\partial$ being each of $\{0\},\{1\}$, or $\{0,1\}$,

$$
\mathcal{S}\left(\left\{\tilde{\phi}_{j, x}^{\text {new }}: x \in I_{j} \backslash \partial\right\}\right) \subset \mathcal{S}\left(\left\{\tilde{\phi}_{j+1, x}^{\text {new }}: x \in I_{j+1} \backslash \partial\right\}\right) .
$$

We will search the modified collections in the form

$$
\tilde{\Phi}_{j}^{\text {new }}=\mathbf{B}_{j} \tilde{\Phi}_{j}, \quad \Phi_{j}^{\text {new }}=\mathbf{B}_{j}^{-T} \Phi_{j},
$$


with, for some vector $\alpha_{j}=\left[\alpha_{j, x}\right]_{x \in I_{j} \backslash\{0,1\}}$ of uniformly bounded scalars with a uniformly bounded number of non-zeros, and $\alpha_{j}^{\uparrow}:=\left[\alpha_{j, 1-x}\right]_{x \in I_{j} \backslash\{0,1\}}$,

$$
\mathbf{B}_{j}:=\begin{array}{|c|c|c|}
\hline 1 & \cdots 0 \cdots & 0 \\
\hline \alpha_{j} & I & \alpha_{j}^{\uparrow} \\
\hline 0 & \cdots 0 \cdots & 1 \\
\hline
\end{array} \text {, and thus } \mathbf{B}_{j}^{-T}=\begin{array}{c|c|c|c|}
\hline 1 & -\alpha_{j}^{T} & 0 \\
\hline: & & I & 0 \\
0 & & & : \\
\hline 0 & -\left(\alpha_{j}^{\uparrow}\right)^{T} & 1 \\
\hline
\end{array}
$$

Since these transformations do not change the spans, and retain $(\underline{\mathcal{D}})$, and $(\underline{\mathcal{L}}),(\underline{\mathcal{S}})$, $(\underline{\mathcal{V}})$, and $(\tilde{\mathcal{L}}),(\tilde{\mathcal{S}})$, the only properties to verify are (2.3) and (2.4).

Under some additional mild conditions, the following theorem shows that suitable coefficients $\alpha_{j, x}$ can always be found.

Theorem 2.2. Assume, for $j \in \mathbb{Z}_{m}$, that $\left\langle\phi_{j, 0}, \tilde{\phi}_{j+1,1}\right\rangle=0$ and

$$
\left\langle\tilde{\phi}_{j, x}, \phi_{j+1,0}\right\rangle=0 \quad \text { for } x \in I_{j} \backslash\{0\},
$$

and that

$$
\sup _{j \in \mathbb{Z}_{m}}\left\langle\phi_{j, 0}, \tilde{\phi}_{j+1,0}\right\rangle\left\langle\tilde{\phi}_{j, 0}, \phi_{j+1,0}\right\rangle<1
$$

Then, with

$$
\alpha_{j+1, x}:=\frac{\left\langle\tilde{\phi}_{j, 0}, \phi_{j+1,0}\right\rangle}{1-\left\langle\phi_{j, 0}, \tilde{\phi}_{j+1,0}\right\rangle\left\langle\tilde{\phi}_{j, 0}, \phi_{j+1,0}\right\rangle}\left\langle\phi_{j, 0}, \tilde{\phi}_{j+1, x}\right\rangle, \quad\left(x \in I_{j+1} \backslash\{0,1\}\right),
$$

we have

$$
\mathcal{S}\left(\tilde{\Phi}_{j}\right) \subset \mathcal{S}\left(\tilde{\Phi}_{j+1}^{\text {new }} \backslash\left\{\tilde{\phi}_{j+1,0}^{\text {new }}, \tilde{\phi}_{j+1,1}^{\text {new }}\right\}\right),
$$

which implies both (2.4) and, after replacing $m$ by $m+1$, by $(\tilde{\mathcal{J}})$ also (2.3).

Before proving this theorem, note that the condition $\left\langle\phi_{j, 0}, \tilde{\phi}_{j+1,1}\right\rangle=0$ follows from $(\underline{\mathcal{L}}),(\tilde{\mathcal{L}})$ when $m$ is sufficiently large. When the dual scaling functions are continuous at $0,(2.7)$ follows from

$$
\tilde{\phi}_{j, x}(0)=0 \text { for } x \in I_{j} \backslash\{0\}, j \in \mathbb{Z}_{m},
$$

which is the analogue of (그 at the dual side, since, by $(\tilde{\mathcal{J}})$, then $\tilde{\phi}_{j, 0}(0) \neq 0$ $\left(j \in \mathbb{Z}_{m}\right)$. A modification of the collections $\Phi_{j}, \tilde{\Phi}_{j}$ from DKU99 such that 2.10) is valid is introduced in DS99a. The condition (2.8) is satisfied when the boundary primal and dual scaling functions on all levels are generated from one pair by means of dyadic dilation, in which case $\left\langle\phi_{j, 0}, \tilde{\phi}_{j+1,0}\right\rangle\left\langle\tilde{\phi}_{j, 0}, \phi_{j+1,0}\right\rangle=\frac{1}{2}$.

Proof. By $(\mathcal{L}),(\tilde{\mathcal{L}})$, the number of nonzero coefficients $\alpha_{j+1, x}$ is uniformly bounded. Because of $(\underline{\mathcal{N}}),(\tilde{\mathcal{N}})$, for $v \in \mathcal{S}\left(\tilde{\Phi}_{j}\right)$ we have

$$
\begin{aligned}
v=\left\langle v, \Phi_{j}\right\rangle \tilde{\Phi}_{j} & =\left\langle v,\left\langle\Phi_{j}, \tilde{\Phi}_{j+1}\right\rangle \Phi_{j+1}\right\rangle\left\langle\tilde{\Phi}_{j}, \Phi_{j+1}\right\rangle \tilde{\Phi}_{j+1} \\
& =\left\langle v, \Phi_{j+1}\right\rangle\left\langle\Phi_{j}, \tilde{\Phi}_{j+1}\right\rangle^{T}\left\langle\tilde{\Phi}_{j}, \Phi_{j+1}\right\rangle \tilde{\Phi}_{j+1},
\end{aligned}
$$

and also

$$
v=\left\langle v, \Phi_{j+1}\right\rangle \tilde{\Phi}_{j+1} .
$$


Comparing the coefficient in front of $\tilde{\phi}_{j+1,0}$ in both expressions for $v$ using (2.7), we infer that

$$
\begin{aligned}
\left(1-\left\langle\phi_{j, 0}, \tilde{\phi}_{j+1,0}\right\rangle\left\langle\tilde{\phi}_{j, 0}, \phi_{j+1,0}\right\rangle\right)\left\langle v, \phi_{j+1,0}\right\rangle & \\
= & \sum_{x \in I_{j+1} \backslash\{0\}}\left\langle\phi_{j, 0}, \tilde{\phi}_{j+1, x}\right\rangle\left\langle\tilde{\phi}_{j, 0}, \phi_{j+1,0}\right\rangle\left\langle v, \phi_{j+1, x}\right\rangle,
\end{aligned}
$$

or, by definition of $\alpha_{j+1, x}$ and the assumption $\left\langle\phi_{j, 0}, \tilde{\phi}_{j+1,1}\right\rangle=0$, that

$$
\left\langle v, \phi_{j+1,0}\right\rangle=\sum_{x \in I_{j+1} \backslash\{0,1\}} \alpha_{j+1, x}\left\langle v, \phi_{j+1, x}\right\rangle,
$$

and so by $(\tilde{\mathcal{S}})$ that $\left\langle v, \phi_{j+1,1}\right\rangle=\sum_{x \in I_{j+1} \backslash\{0,1\}} \alpha_{j+1,1-x}\left\langle v, \phi_{j+1, x}\right\rangle$. By substituting these expressions into (2.11), by definition of $\tilde{\Phi}_{j+1}^{\text {new }}$ we find that

$$
v=\sum_{x \in I_{j+1} \backslash\{0,1\}}\left\langle v, \phi_{j+1, x}\right\rangle \tilde{\phi}_{j+1, x}^{\text {new }} \quad\left(=\sum_{x \in I_{j+1} \backslash\{0,1\}}\left\langle v, \phi_{j+1, x}^{\text {new }}\right\rangle \tilde{\phi}_{j+1, x}^{\text {new }}\right),
$$

which completes the proof.

In Theorem 2.2 the vector $\alpha_{j}$ was constructed such that (2.9) is valid, the property of which is generally stronger than the combination of (2.3) and (2.4), and therefore generally requires more nonzero coefficients than is strictly necessary, resulting in modified scaling functions with larger supports. In the following theorem, under some additional conditions, however without (2.7), we present a construction that realizes (2.3) and (2.4) with a generally minimal number of nonzero coefficients $\alpha_{j, x}$.

Theorem 2.3. For $j \in \mathbb{Z}_{m}$, let $\bar{I}_{j} \subset I_{j} \backslash\{0,1\}$ with $\# \bar{I}_{j}=\tilde{d}$, $\sup \left\{x: x \in \bar{I}_{j}\right\} \lesssim 2^{-j}$, and

$$
\inf _{0 \neq p \in P_{\tilde{d}-1}(0,1)} \sup _{0 \neq v \in \mathcal{S}\left(\left\{\phi_{j, x}: x \in \bar{I}_{j}\right\}\right)} \frac{|\langle p, v\rangle|}{\|p\|\|v\|} \gtrsim 1 .
$$

With $Q_{j}: L_{2}(0,1) \rightarrow \mathcal{S}\left(\left\{\phi_{j, x}: x \in \bar{I}_{j}\right\}\right)$ being the projector with $\Im\left(I-Q_{j}\right) \perp_{L_{2}(0,1)}$ $P_{\tilde{d}-1}(0,1)$, whose existence follows from (2.12), let

$$
\left\langle\left(I-Q_{j+1}\right) \phi_{j+1,0}, \tilde{\phi}_{j, x}\right\rangle=0 \quad \text { for all } x \in I_{j} \backslash\left(\bar{I}_{j}+\{0\}\right) .
$$

Then for each $j \in \mathbb{Z}_{m}$, there exists a unique vector $\alpha_{j}=\left[\alpha_{j, x}\right]_{x \in I_{j} \backslash\{0,1\}}$ with $\alpha_{j, x}=0$ when $x \notin \bar{I}_{j}$, whose coefficients are uniformly bounded, such that $\Phi_{j}^{\text {new }}$ and $\tilde{\Phi}_{j}^{\text {new }}$ defined by (2.5), (2.6) satisfy (2.3) and (2.4). The coefficients $\left\{\alpha_{j, x}: x \in \bar{I}_{j}\right\}$ can be found by solving

$$
\phi_{j, 0}^{\text {new }}:=\phi_{j, 0}+\sum_{x \in \bar{I}_{j}} \alpha_{j, x} \phi_{j, x} \perp_{L_{2}(0,1)} P_{\tilde{d}-1}(0,1) .
$$

Proof. After selecting a basis $\left\{p_{0}, \ldots, p_{\tilde{d}-1}\right\}$ for $P_{\tilde{d}-1}(0,1)$, solving (2.14) amounts to inverting the matrix $\left[\left\langle\phi_{j, x}, p_{\ell}\right\rangle\right]_{x \in \bar{I}_{j}, 0 \leq \ell \leq \tilde{d}-1}$. Because of (2.12), there is a unique, uniformly bounded solution. Note that $\phi_{j, 0}^{\text {new }}=\left(I-Q_{j}\right) \phi_{j, 0}$.

Knowing $P_{\tilde{d}-1}(0,1) \subset \mathcal{S}\left(\tilde{\Phi}_{j}\right)=\mathcal{S}\left(\tilde{\Phi}_{j}^{\text {new }}\right)$, (S) and (D) $(2.3)$ is equivalent to $\phi_{j, 0}^{\text {new }} \perp_{L_{2}(0,1)} P_{\tilde{d}-1}(0,1)$, which holds by construction.

Knowing $\mathcal{S}\left(\tilde{\Phi}_{j}^{\text {new }}\right) \subset \mathcal{S}\left(\tilde{\Phi}_{j+1}^{\text {new }}\right),(\underline{\mathcal{S}}),(\tilde{\mathcal{S}})$ and $(\bar{D}),(2.4)$ is equivalent to

$$
\phi_{j+1,0}^{\text {new }} \perp_{L_{2}(0,1)} \mathcal{S}\left(\tilde{\Phi}_{j}^{\text {new }} \backslash\left\{\tilde{\phi}_{j, 0}^{\text {new }}\right\}\right) \text {. }
$$


By construction, for $x \in I_{j} \backslash\left(\bar{I}_{j}+\{0\}\right)$ we have $\tilde{\phi}_{j, x}^{\text {new }} \subset \mathcal{S}\left(\left\{\tilde{\phi}_{j, x}: x \in I_{j} \backslash\left(\bar{I}_{j}+\{0\}\right)\right\}\right)$, and so the assumption (2.13) shows that

$$
\left\langle\phi_{j+1,0}^{\text {new }}, \tilde{\phi}_{j, x}^{\text {new }}\right\rangle=0 \quad \text { for all } x \in I_{j} \backslash\left(\bar{I}_{j} \cup\{0\}\right) .
$$

From (2.3) we have $p_{\ell}=\sum_{x \in I_{j} \backslash\{0,1\}}\left\langle p_{\ell}, \phi_{j, x}^{\text {new }}\right\rangle \tilde{\phi}_{j, x}^{\text {new }}$ for $0 \leq \ell \leq \tilde{d}-1$. From (2.16) and $\phi_{j+1,0}^{\text {new }} \perp_{L_{2}(0,1)} P_{\tilde{d}-1}(0,1)$, we infer that $\phi_{j+1,0}^{\text {new }} \perp_{L_{2}(0,1)} \sum_{x \in \bar{I}_{j}}\left\langle p_{\ell}, \phi_{j, x}^{\text {new }}\right\rangle \tilde{\phi}_{j, x}^{\text {new }}$. Since $\phi_{j, x}^{\text {new }}=\phi_{j, x}$ for $x \in I_{j} \backslash\{0,1\}$, and $\left[\left\langle p_{\ell}, \phi_{j, x}\right\rangle\right]_{0 \leq \ell \leq \tilde{d}-1, x \in \bar{I}_{j}}$ is an invertible system because of (2.12), this means that $\left\langle\phi_{j+1,0}^{\text {new }}, \tilde{\phi}_{j, x}^{\text {new }}\right\rangle=0$ also for $x \in \bar{I}_{j}$, which completes the proof of (2.15) and thus of the theorem.

In the following, for a particular choice of $\bar{I}_{j}$, we verify the conditions of Theorem 2.3 for any pair of biorthogonal scaling functions constructed in DKU99. By definition and $(\mathcal{V}), \tilde{\phi}_{j, 0}$ is the dual of the unique primal scaling function $\phi_{j, 0}$ that does not vanish at 0 . We now take $\bar{I}_{j}$ to be the index set of those $\tilde{d}$ dual scaling functions other than $\tilde{\phi}_{j, 0}$ whose supports have their minima closest to 0 . Inside this collection, there is one $\tilde{\phi}_{j, z}$ such that $\left(\phi_{j, z}, \tilde{\phi}_{j, z}\right)$ is a biorthogonal pair of scaling functions on the line as constructed in [CDF92]. The set $I_{j}^{\circ}:=\{0\}+\bar{I}_{j} \backslash\{z\}$ (denoted as $\tilde{I}_{j}^{\mathrm{L}}$ in DKU99) is the index set of the left boundary adapted scaling functions. By construction of the scaling functions in DKU99, it holds that $\left\langle\phi_{j+1, y}, \tilde{\phi}_{j, x}\right\rangle=0$ for all $y \in \bar{I}_{j+1}, x \in I_{j} \backslash\left(\bar{I}_{j}+\{0\}\right)$, so that (2.13) is valid.

Since all $\left\{\phi_{j, x}: x \in \bar{I}_{j}\right\}$ are constructed from one set by means of dyadic dilation, the quantity on the left-hand side of (2.12) is independent of $j$. By construction of the boundary adapted scaling functions in [DKU99], there exists a basis $\left\{p_{x}: x \in\right.$ $\left.I_{j}^{\circ}\right\}$ for $P_{\tilde{d}-1}(0,1)$ such that

$$
p_{x}=\tilde{\phi}_{j, x}+\sum_{v \in I_{j} \backslash I_{j}^{\circ}}\left\langle p_{x}, \phi_{j, v}\right\rangle \tilde{\phi}_{j, v} .
$$

By applying this basis, using biorthogonality we infer that 2.12) is equivalent to invertibility of $\left[\left\langle\tilde{\phi}_{j, x}+\left\langle p_{x}, \phi_{j, z}\right\rangle \tilde{\phi}_{j, z}, \phi_{j, y}\right\rangle\right]_{x \in I_{j}^{\circ}, y \in \bar{I}_{j}}$. Since, modulo invertible transformations, $\left\{\tilde{\phi}_{j, x}+\left\langle p_{x}, \phi_{j, z}\right\rangle \tilde{\phi}_{j, z}: x \in I_{j}^{\circ}\right\}$ is the collection of left boundary adapted dual scaling functions that one obtains by increasing the parameter $r$ from (2.2) by 1 , and $\left\{\phi_{j, y}: y \in \bar{I}_{j}\right\}$ is the collection of left boundary adapted primal scaling functions from which $\phi_{j, 0}$ is removed, and to which the interior primal scaling function succeeding $\phi_{j, z}$ is added, Theorem 4.2 of DS98] shows that the aforementioned matrix is indeed invertible, and thus that (2.12) is valid.

Remark 2.4. For the collections $\Phi_{j}^{\text {new }}, \tilde{\Phi}_{j}^{\text {new }}$ yielded by Theorem 2.3, it holds that the functions from $\left\{\phi_{j, x}^{\text {new }}: x \in I_{j} \backslash\{0,1\}\right\}$ vanish at $\{0,1\}$, and that $P_{\tilde{d}-1}(0,1) \subset$ $\mathcal{S}\left(\left\{\tilde{\phi}_{j, x}^{\text {new }}: x \in I_{j} \backslash\{0,1\}\right\}\right)$, meaning that these reduced collections satisfy so-called complementary boundary conditions of order zero. Starting with any pair $\left(\Phi_{j}\right)_{j}$, $\left(\tilde{\Phi}_{j}\right)_{j}$ from DKU99, one may verify that these reduced collections, if not equal, span the same spaces as the collections with zeroth order complementary boundary conditions constructed in DS98. In this paper, however, the full collections $\Phi_{j}^{\text {new }}$ and $\tilde{\Phi}_{j}^{\text {new }}$ will be needed. Under appropriate conditions, transformations as in Theorems 2.2 or 2.3 can be applied repeatedly, starting with $\left\{\phi_{j, x}^{\text {new }}: x \in I_{j} \backslash\{0,1\}\right\}$ and $\left\{\tilde{\phi}_{j, x}^{\text {new }}: x \in I_{j} \backslash\{0,1\}\right\}$, yielding biorthogonal collections with complementary boundary conditions of higher order. 
As a final ingredient on $[0,1]$, we define collections of functionals $\Lambda_{j}=\left[\lambda_{j, x}\right]_{x \in I_{j}}$ $\subset C[0,1]^{\prime}, \tilde{\Lambda}_{j}=\left[\tilde{\lambda}_{j, x}\right]_{x \in I_{j}} \subset L_{2}(0,1)^{\prime}$ by $\lambda_{j, 0}(v)=v(0), \lambda_{j, 1}(v)=v(1), \tilde{\lambda}_{j, 0}(v)$ $=\tilde{\lambda}_{j, 1}(v)=0$, and, for $x \in I_{j} \backslash\{0,1\}, \lambda_{j, x}(v)=\left\langle v, \tilde{\phi}_{j, x}^{\text {new }}\right\rangle$ and $\tilde{\lambda}_{j, x}(v)=\left\langle v, \phi_{j, x}^{\text {new }}\right\rangle$. Note that, with the common notation as discussed at the end of Section 1 .

$$
\left\langle\Phi_{j}^{\text {new }}, \Lambda_{j}\right\rangle=I, \quad\left\langle\tilde{\Phi}_{j}^{\text {new }}, \tilde{\Lambda}_{j}\right\rangle=\operatorname{diag}([0,1, \ldots, 1,0]) .
$$

In the next two sections, we will drop the superscript "new", so that with $\Phi_{j}$ and $\tilde{\Phi}_{j}$ collections are meant, as constructed in Theorems 2.2 or 2.3 , that satisfy $(\mathcal{D}),(\mathcal{L})-(\mathcal{V})$ or $(\tilde{\mathcal{L}})-(\tilde{\mathcal{B}})$, respectively, and, in addition, (2.3) and (2.4). Using these collections, we will construct composite wavelet bases on general domains or manifolds following the construction introduced in DS99a. We will present the main steps, and refer to DS99a] or [Ste04a] for proofs.

\section{Biorthogonal multiresolution on $(0,1)^{n}$}

With $\square:=(0,1)^{n}$, let $\langle\cdot, \cdot\rangle=\langle\cdot, \cdot\rangle_{L_{2}(\square)}$ throughout this section. With $\Pi$ we will denote the collection of all affine mappings from $\square$ onto $\square$, which consists of any composition of reflections of type $x \mapsto\left(x_{1}, \ldots, x_{i-1}, 1-x_{i}, x_{i+1}, \ldots, x_{n}\right)(1 \leq i \leq n)$ and permutations of the $n$ Cartesian coordinates. With a face of $\square$, we mean a complete, closed face of any dimension $0 \leq k \leq n-1$, i.e., for $n=3$, it is either a vertex, an edge, or a facet.

For $j \in \mathbb{N}_{0}$, we define index sets

$$
I_{j}^{\square}=\left(I_{j}\right)^{n}, \quad J_{j}^{\square}=\left(J_{j} \cup I_{j}\right)^{n} \backslash I_{j}^{\square} ;
$$

collections of functions

$$
\Phi_{j}^{\square}=\left[\phi_{j, x}^{\square}\right]_{x \in I_{j}^{\square}}, \quad \tilde{\Phi}_{j}^{\square}=\left[\tilde{\phi}_{j, x}^{\square}\right]_{x \in I_{j}^{\square}}, \quad \text { and } \quad \Xi_{j, x}^{\square}=\left[\xi_{j, x}^{\square}\right]_{x \in J_{j}^{\square}},
$$

by

$$
\phi_{j, x}^{\square}(y)=\prod_{i=1}^{n} \phi_{j, x_{i}}\left(y_{i}\right), \quad \tilde{\phi}_{j, x}^{\square}(y)=\prod_{i=1}^{n} \tilde{\phi}_{j, x_{i}}\left(y_{i}\right),
$$

and

$$
\xi_{j, x}^{\square}(y)=\prod_{i=1}^{n} \omega_{j, x_{i}}\left(y_{i}\right) \quad \text { with } \quad \omega_{j, x_{i}}:=\xi_{j, x_{i}}
$$

when $x_{i} \in J_{j}$ and $\omega_{j, x_{i}}:=\phi_{j, x_{i}}$ otherwise; and collections of functionals

$$
\Lambda_{j}^{\square}=\left[\lambda_{j, x}\right]_{j \in I_{j}^{\square}} \subset C(\bar{\square})^{\prime}, \quad \tilde{\Lambda}_{j}^{\square}=\left[\tilde{\lambda}_{j, x}\right]_{j \in I_{j}^{\square}} \subset L_{2}(\square)^{\prime}
$$

by, for univariate functions, $v_{i}$,

$$
\lambda_{j, x}^{\square}\left(\prod_{i} v_{i}\right)=\prod_{i} \lambda_{j, x_{i}}\left(v_{i}\right), \quad \tilde{\lambda}_{j, x}^{\square}\left(\prod_{i} v_{i}\right)=\prod_{i} \tilde{\lambda}_{j, x_{i}}\left(v_{i}\right),
$$

which determines $\lambda_{j, x}^{\square}, \tilde{\lambda}_{j, x}^{\square}$ on the whole of $C(\bar{\square}), L_{2}(\square)$, respectively. Clearly, we have

$$
\left\langle\Phi_{j}^{\square}, \tilde{\Phi}_{j}^{\square}\right\rangle=I, \quad\left\langle\Phi_{j}^{\square}, \Lambda_{j}^{\square}\right\rangle=I, \quad \text { and } \quad\left\langle\tilde{\Phi}_{j}^{\square}, \tilde{\Lambda}_{j}^{\square}\right\rangle=\operatorname{diag}\left(\left\{d_{j, x}: x \in I_{j}^{\square}\right\}\right)
$$

with $d_{j, x}=0$ if $x \in \partial \square$, and $d_{j, x}=1$, otherwise.

For a proof of the next proposition, we refer to DS99a, Proposition 3.1], where for the projector at the dual side (2.3) has to be used. 
Proposition 3.1. For the projectors $P_{j}^{\square}: v \mapsto\left\langle v, \Lambda_{j}^{\square}\right\rangle \Phi_{j}^{\square}, \tilde{P}_{j}^{\square}: v \mapsto\left\langle v, \tilde{\Lambda}_{j}^{\square}\right\rangle \tilde{\Phi}_{j}^{\square}$ onto $\mathcal{S}\left(\Phi_{j}^{\square}\right)$ and to $\mathcal{S}\left(\tilde{\Phi}_{j}^{\square}\right)$, respectively, we have

$$
\left\|\left(I-\tilde{P}_{j}^{\square}\right) v\right\|_{L_{2}(\square)} \lesssim 2^{-\tilde{d} j}|v|_{H^{\tilde{d}}(\square)} \quad\left(v \in H^{\tilde{d}(\square)),}\right.
$$

and, when $d>\frac{n}{2}$,

$$
\left\|\left(I-P_{j}^{\square}\right) v\right\|_{L_{2}(\square)} \lesssim 2^{-d j}|v|_{H^{d}(\square)} \quad\left(v \in H^{d}(\square)\right) .
$$

Moreover, writing $P_{j}^{\square} v=\sum_{x \in I_{j}^{\square}} \mathbf{c}_{j, x} \phi_{j, x}^{\square}, \tilde{P}_{j}^{\square} v=\sum_{x \in I_{j}^{\square}} \tilde{\mathbf{c}}_{j, x} \tilde{\phi}_{j, x}^{\square}$, it holds that if $v$ vanishes on a face of $\square$, then $\mathbf{c}_{j, x}=0$ for all $x$ on that face, whereas $\tilde{\mathbf{c}}_{j, x}=0$ for all $x \in \partial \square$ anyway.

Remark 3.2. The (primal and dual) wavelets that we are going to construct on general domains or manifolds $\Omega$ will depend on $\mathcal{S}\left(\Phi_{j}^{\square}\right)$ and $\mathcal{S}\left(\tilde{\Phi}_{j}^{\square}\right)$, but not on the selection of their bases $\Phi_{j}^{\square}$ and $\tilde{\Phi}_{j}^{\square}$. On the other hand, they do depend on the collection $\Xi_{j}^{\square}$. We have constructed this collection by taking tensor products of functions from $\Phi_{j}$ and $\Xi_{j}$. However, $\Xi_{j}^{\square}=\left\{\xi_{j, x}^{\square}: x \in J_{j}^{\square}\right\}$ can be any collection that satisfies $\xi_{j, x}^{\square}(y)=0$ if not $|x-y| \lesssim 2^{-j} ; \xi_{j, x}^{\square}$ vanishes on any face of $\square$ that does not contain $x ;\left.\left(\xi_{j, x}^{\square}\right)\right|_{\partial \square}=\left.\left(\xi_{j, \pi(x)}^{\square} \circ \pi\right)\right|_{\partial \square}$ for all $\pi \in \Pi ; \Upsilon_{j+1}^{\square}:=\left[\begin{array}{l}\left(\Phi_{j}^{\square}\right)^{T} \quad\left(\Xi_{j}^{\square}\right)^{T}\end{array}\right]^{T}$ is a uniform $L_{2}(\square)$-Riesz basis for $\mathcal{S}\left(\Phi_{j+1}^{\square}\right)$; and, but only if one is interested in having dual wavelets that are also locally supported, for which $\left\langle\Upsilon_{j+1}^{\square}, \tilde{\Phi}_{j+1}^{\square}\right\rangle^{-1}$ is uniformly local. Taking an alternative collection $\Xi_{j}^{\square}$ can yield wavelets with smaller supports, or, which still has to be investigated, it might result in quantitatively better conditioned wavelet bases.

\section{Biorthogonal wavelets on $\Omega$}

4.1. Setting. For some $n^{\prime} \geq n \geq 1$, let $\Omega$ be a $n$-dimensional bounded manifold in $\mathbb{R}^{n^{\prime}}$, with or without a boundary. We assume that $\Omega$ is given as

$$
\bar{\Omega}=\bigcup_{q=1}^{M} \overline{\Omega_{q}} \text {, with } \Omega_{q} \cap \Omega_{q^{\prime}}=\emptyset \text { when } q \neq q^{\prime}, \text { and } \Omega_{q}=\kappa_{q}(\square),
$$

where $\kappa_{q}: \mathbb{R}^{n} \rightarrow \mathbb{R}^{n^{\prime}}$ are some smooth, regular parametrizations. We assume that the splitting of $\Omega$ into the patches $\Omega_{q}$ is conforming in the sense that for any $q \neq q^{\prime}$, $\overline{\Omega_{q}} \cap \overline{\Omega_{q}^{\prime}}$ is either empty or

$$
\kappa_{q}^{-1}\left(\overline{\Omega_{q}} \cap \overline{\Omega_{q}^{\prime}}\right) \text { is a face of } \square,
$$

and, in addition, that the parametrizations can be chosen such that the following matching condition is satisfied: there exists a $\pi \in \Pi$ with

$$
\kappa_{q^{\prime}} \circ \pi \circ \kappa_{q}^{-1}=I \quad \text { on } \overline{\Omega_{q}} \cap \overline{\Omega_{q}^{\prime}} \text {. }
$$

Note that our setting allows $\Omega$ to be a bounded domain in $\mathbb{R}^{n}$, as well as an open or closed bounded manifold in $\mathbb{R}^{n^{\prime}}$ for some $n^{\prime}>n$.

We equip $L_{2}(\Omega)$ with the modified $L_{2}(\Omega)$-scalar product

$$
\langle\langle u, v\rangle\rangle:=\sum_{q=1}^{M}\left\langle u \circ \kappa_{q}, v \circ \kappa_{q}\right\rangle_{L_{2}(\square)},
$$

which defines a norm that is equivalent to the canonical $L_{2}(\Omega)$-norm. 
We include the possibility that homogeneous, zeroth order Dirichlet boundary conditions are prescribed on some part $\partial \Omega_{D} \subset \bar{\Omega} \backslash \Omega$, for which, for all $1 \leq q \leq M$,

$$
\kappa_{q}^{-1}\left(\partial \Omega_{D} \cap \bar{\Omega}_{q}\right) \text { is a (possibly empty) union of faces of } \square \text {. }
$$

4.2. Biorthogonal multiresolution on $\Omega$. We define the index sets $I_{j}^{\Omega} \subset \bar{\Omega} \backslash \partial \Omega_{D}$, and analogously $J_{j}^{\Omega}$, by

$$
I_{j}^{\Omega}=\left(\bigcup_{q=1}^{M} \kappa_{q}\left(I_{j}^{\square}\right)\right) \cap\left(\bar{\Omega} \backslash \partial \Omega_{D}\right) .
$$

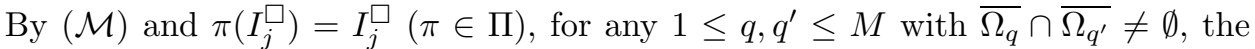
sets $\kappa_{q}\left(I_{j}^{\square}\right)$ and $\kappa_{q^{\prime}}\left(I_{j}^{\square}\right)$ restricted to this interface coincide.

Setting, for $x \in \bar{\Omega}$,

$$
k(x)=\#\left\{q: x \in \overline{\Omega_{q}}\right\},
$$

for $j \in \mathbb{N}_{0}$ we define the collections $\Phi_{j}^{\Omega}=\left[\phi_{j, x}^{\Omega}\right]_{x \in I_{j}^{\Omega}}, \tilde{\Phi}_{j}^{\Omega}=\left[\tilde{\phi}_{j, x}^{\Omega}\right]_{x \in I_{j}^{\Omega}}, \Xi_{j}^{\Omega}=$ $\left[\xi_{j, x}^{\Omega}\right]_{x \in J_{j}^{\Omega}}$, and $\Upsilon_{j+1}^{\Omega}:=\left[\begin{array}{ll}\left(\Phi_{j}^{\Omega}\right)^{T} & \left(\Xi_{j}^{\Omega}\right)^{T}\end{array}\right]^{T}$ of functions on $\Omega$ by

$$
\phi_{j, x}^{\Omega}(y)=\frac{1}{\sqrt{k(x)}} \cdot\left\{\begin{array}{cl}
\phi_{j, \kappa_{q}^{-1}(x)}^{\square}\left(\kappa_{q}^{-1}(y)\right) & \begin{array}{l}
\text { when } x \in \overline{\Omega_{q}} \\
0
\end{array}, y \in \Omega_{q} \text { for a } 1 \leq q \leq M, \\
\text { elsewhere, }
\end{array}\right.
$$

with analogous definitions of $\tilde{\phi}_{j, x}^{\Omega}$ and $\xi_{j, x}^{\Omega}$. Note that by (ㄱ) $(\mathcal{V})$ and (4.2), $\phi_{j, x}$ and $\xi_{j, x}$ extend to continuous functions on $\bar{\Omega}$ that vanish on $\partial \Omega_{D}$. However, since $\tilde{\Phi}_{j}$ does not satisfy the analogue of (V) at the dual side, the collection $\left\{\tilde{\phi}_{j, x}^{\Omega}: x \in\right.$ $\left.\bigcup_{q=1}^{M} \kappa_{q}\left(I_{j}^{\square} \square\right)\right\} \subset \tilde{\Phi}_{j}^{\Omega}$, that is, the collection of dual scaling functions that are not 'glued' over the patch interfaces, contains functions that do not vanish on all interfaces, and which thus are discontinuous, as well as functions that do not vanish on $\partial \Omega_{D}$. By assumptions $(\mathcal{L})$ or $(\tilde{\mathcal{L}})$, the collections $\Phi_{j}^{\Omega}, \tilde{\Phi}_{j}^{\Omega}, \Xi_{j}^{\Omega}$ are uniformly local. By this we mean that $x \in \operatorname{supp} \phi_{j, x}$, and that $d_{\Omega}(x, y) \lesssim 2^{-j}$ for any $y \in \operatorname{supp} \phi_{j, x}$, where $d_{\Omega}(x, y)$ denotes the geodesic distance of $x$ and $y$ over $\Omega$, i.e., the length of the shortest curve on $\Omega$ connecting $x$ and $y$.

Setting $\mathbf{E}_{j, q}: \ell_{2}\left(I_{j}^{\square}\right) \rightarrow \ell_{2}\left(I_{j}^{\Omega}\right)$, and analogously $\mathbf{F}_{j, q}: \ell_{2}\left(J_{j}^{\square}\right) \rightarrow \ell_{2}\left(J_{j}^{\Omega}\right)$, by

$$
\left(\mathbf{E}_{j, q} \mathbf{c}_{j}^{\square}\right)_{x}=\frac{1}{\sqrt{k(x)}} \cdot\left\{\begin{array}{cl}
\mathbf{c}_{j, \kappa_{q}^{-1}(x)}^{\square} & x \in \overline{\Omega_{q}}, \\
0 & \text { otherwise, }
\end{array}\right.
$$

by construction of $\Phi_{j}^{\Omega}$ from $\Phi_{j}^{\square}$, and the fact that the latter collection is a uniform $L_{2}(\square)$-Riesz system, we have

$$
\left\langle\left\langle\Phi_{j}^{\Omega}, \Phi_{j}^{\Omega}\right\rangle\right\rangle=\sum_{q=1}^{M} \mathbf{E}_{j, q}\left\langle\Phi_{j}^{\square}, \Phi_{j}^{\square}\right\rangle_{L_{2}(\square)} \mathbf{E}_{j, q}^{T} \approx \sum_{q=1}^{M} \mathbf{E}_{j, q} \mathbf{E}_{j, q}^{T}=I,
$$

meaning that $\Phi_{j}^{\Omega}$, and analogously $\tilde{\Phi}_{j}^{\Omega}$ and $\Upsilon_{j}^{\Omega}$, are uniform $L_{2}(\Omega)$-Riesz systems. Moreover, we have biorthogonality, i.e.,

$$
\left\langle\left\langle\Phi_{j}^{\Omega}, \tilde{\Phi}_{j}^{\Omega}\right\rangle\right\rangle=\sum_{q=1}^{M} \mathbf{E}_{j, q}\left\langle\Phi_{j}^{\square}, \tilde{\Phi}_{j}^{\square}\right\rangle_{L_{2}(\square)} \mathbf{E}_{j, q}^{T}=\sum_{q=1}^{M} \mathbf{E}_{j, q} \mathbf{E}_{j, q}^{T}=I .
$$


Setting $\Lambda_{j}^{\Omega}=\left[\lambda_{j, x}^{\Omega}\right]_{x \in I_{j}^{\Omega}}, \tilde{\Lambda}_{j}^{\Omega}=\left[\tilde{\lambda}_{j, x}^{\Omega}\right]_{x \in I_{j}^{\Omega}}$ by

$$
\lambda_{j, x}^{\Omega}(u)=\sqrt{k(x)} \lambda_{j, \kappa_{q}^{-1}(x)}^{\square}\left(u \circ \kappa_{q}\right) \quad \text { when } x \in \bar{\Omega}_{q},
$$

with an analogous definition of $\tilde{\lambda}_{j, x}^{\Omega}$, for $P_{j}^{\Omega}: C(\bar{\Omega}) \rightarrow \mathcal{S}\left(\Phi_{j}^{\Omega}\right): v \mapsto\left\langle\left\langle v, \Lambda_{j}^{\Omega}\right\rangle\right\rangle \Phi_{j}^{\Omega}$ we have

$$
\left(\left(I-P_{j}^{\Omega}\right) v\right) \circ \kappa_{q}=\left(I-P_{j}^{\square}\right)\left(v \circ \kappa_{q}\right), \quad(1 \leq q \leq M),
$$

with the analogous statement for $\tilde{P}_{j}^{\Omega}: L_{2}(\Omega) \rightarrow \mathcal{S}\left(\tilde{\Phi}_{j}^{\Omega}\right): v \mapsto\left\langle\left\langle v, \Lambda_{j}^{\Omega}\right\rangle\right\rangle \Phi_{j}^{\Omega}$.

Because of (ㄱ) , at the primal side we have the analogue of (2.3), i.e., for $\partial$ being each of $\{0\},\{1\}$, or $\{0,1\}$, it holds that

$$
\mathcal{S}\left(\left\{\phi_{j, x}: x \in I_{j} \backslash \partial\right\}\right) \subset \mathcal{S}\left(\left\{\phi_{j+1, x}: x \in I_{j+1} \backslash \partial\right\}\right),
$$

and also

$$
\mathcal{S}\left(\left\{v_{j+1, x}: x \in\left(I_{j}+J_{j}\right) \backslash \partial\right\}\right)=\mathcal{S}\left(\left\{\phi_{j+1, x}: x \in I_{j+1} \backslash \partial\right\}\right) .
$$

As a consequence, despite the possible exclusion of functions because of the boundary conditions (cf. (4.3)), we have

$$
\mathcal{S}\left(\Phi_{j}^{\Omega}\right) \subset \mathcal{S}\left(\Phi_{j+1}^{\Omega}\right), \quad \mathcal{S}\left(\tilde{\Phi}_{j}^{\Omega}\right) \subset \mathcal{S}\left(\tilde{\Phi}_{j+1}^{\Omega}\right), \quad \text { and } \quad \mathcal{S}\left(\Upsilon_{j+1}^{\Omega}\right)=\mathcal{S}\left(\Phi_{j+1}^{\Omega}\right)
$$

In particular, the representations of the inclusions (the "refinement matrices") or the basis transformations can be expressed in terms of their counterparts on the cube, and with that in terms of their counterparts on the interval (however, cf. Remark 3.2), as follows:

$$
\begin{aligned}
& \left\langle\left\langle\Phi_{j}^{\Omega}, \tilde{\Phi}_{j+1}^{\Omega}\right\rangle\right\rangle^{T}=\sum_{q=1}^{M} \mathbf{E}_{j+1, q}\left\langle\Phi_{j}^{\square}, \tilde{\Phi}_{j+1}^{\square}\right\rangle_{L_{2}(\square)}^{T} \mathbf{E}_{j, q}^{T}, \\
& \left\langle\left\langle\tilde{\Phi}_{j}^{\Omega}, \Phi_{j+1}^{\Omega}\right\rangle\right\rangle^{T}=\sum_{q=1}^{M} \mathbf{E}_{j+1, q}\left\langle\tilde{\Phi}_{j}^{\square}, \Phi_{j+1}^{\square}\right\rangle_{L_{2}(\square)}^{T} \mathbf{E}_{j, q}^{T}, \\
& \left\langle\left\langle\Upsilon_{j+1}^{\Omega}, \tilde{\Phi}_{j+1}^{\Omega}\right\rangle\right\rangle^{T}=\sum_{q=1}^{M} \mathbf{E}_{j+1, q}\left\langle\Upsilon_{j+1}^{\square}, \tilde{\Phi}_{j+1}^{\square}\right\rangle_{L_{2}(\square)}^{T}\left[\begin{array}{c|c}
\mathbf{E}_{j, q}^{T} & 0 \\
\hline 0 & \mathbf{F}_{j, q}^{T}
\end{array}\right], \\
& \left\langle\left\langle\Upsilon_{j+1}^{\Omega}, \tilde{\Phi}_{j+1}^{\Omega}\right\rangle\right\rangle^{-T}=\sum_{q=1}^{M}\left[\begin{array}{c|c}
\mathbf{E}_{j, q} & 0 \\
\hline 0 & \mathbf{F}_{j, q}
\end{array}\right]\left\langle\Upsilon_{j+1}^{\square}, \tilde{\Phi}_{j+1}^{\square}\right\rangle_{L_{2}(\square)}^{-T} \mathbf{E}_{j+1, q}^{T},
\end{aligned}
$$

where, because of (2.1), as the other three matrices, $\left\langle\left\langle\Upsilon_{j+1}^{\Omega}, \tilde{\Phi}_{j+1}^{\Omega}\right\rangle\right\rangle^{-T}$ is uniformly local, meaning that only entries indexed by $(x, y)$ with $d_{\Omega}(x, y) \lesssim 2^{-j}$ might be nonzero.

4.3. Wavelets. Since $\Phi_{j}^{\Omega}, \tilde{\Phi}_{j}^{\Omega}$ are biorthogonal, uniformly local, uniform $L_{2}(\Omega)$ Riesz systems, the collections of primal and dual wavelets $\Psi_{j}^{\Omega}, \tilde{\Psi}_{j}^{\Omega}$ defined by

$$
\Psi_{j}^{\Omega}=\Xi_{j}^{\Omega}-\left\langle\left\langle\Xi_{j}^{\Omega}, \tilde{\Phi}_{j}^{\Omega}\right\rangle\right\rangle \Phi_{j}^{\Omega}, \quad \tilde{\Psi}_{j}^{\Omega}=[0 \mid I]\left\langle\left\langle\Upsilon_{j+1}^{\Omega}, \tilde{\Phi}_{j+1}^{\Omega}\right\rangle\right\rangle^{-T} \tilde{\Phi}_{j+1}^{\Omega}
$$

are biorthogonal (with respect to $\langle\langle\cdot, \cdot\rangle\rangle$ ), uniformly local, uniform $L_{2}(\Omega)$-Riesz bases

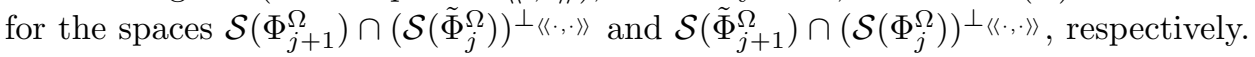


Here also $\left\langle\left\langle\Xi_{j}^{\Omega}, \tilde{\Phi}_{j}^{\Omega}\right\rangle\right\rangle$ can be expressed in terms of its counterpart on the cube, via

$$
\begin{aligned}
\left\langle\left\langle\Xi_{j}^{\Omega}, \tilde{\Phi}_{j}^{\Omega}\right\rangle\right\rangle & =\sum_{q=1}^{M} \mathbf{F}_{j, q}\left\langle\Xi_{j}^{\square}, \tilde{\Phi}_{j}^{\square}\right\rangle_{L_{2}(\square)} \mathbf{E}_{j, q}^{T} \\
& =\sum_{q=1}^{M} \mathbf{F}_{j, q}\left\langle\Xi_{j}^{\square}, \tilde{\Phi}_{j+1}^{\square}\right\rangle_{L_{2}(\square)}\left\langle\tilde{\Phi}_{j}^{\square}, \Phi_{j+1}^{\square}\right\rangle_{L_{2}(\square)}^{T} \mathbf{E}_{j, q}^{T} .
\end{aligned}
$$

For $s \geq 0$, we set

$$
\mathcal{H}^{s}(\Omega)=\left\{\begin{array}{cc}
H_{0, \partial \Omega_{D}}^{s}(\Omega) & \text { when } s \leq 1, \\
H^{s}(\Omega) \cap H_{0, \partial \Omega_{D}}^{1}(\Omega) & \text { when } s>1,
\end{array} \quad \tilde{\mathcal{H}}^{s}(\Omega)=H^{s}(\Omega),\right.
$$

$\mathcal{H}^{-s}(\Omega)=\tilde{\mathcal{H}}^{s}(\Omega)^{\prime}$, and $\tilde{\mathcal{H}}^{-s}(\Omega)=\mathcal{H}^{s}(\Omega)^{\prime}$, where, in the true manifold case, we restrict ourselves to those $s$ for which the definition of the Sobolev spaces is permitted by the regularity of the manifold. Furthermore, we set $\Psi_{-1}^{\Omega}=\Phi_{0}^{\Omega}$ and $\tilde{\Psi}_{-1}^{\Omega}=\tilde{\Phi}_{0}^{\Omega}$. Then, together, the nesting of primal and dual spaces, the fact that they can be equipped with biorthogonal uniform $L_{2}(\Omega)$-Riesz bases, the Jackson estimates that can be deduced from Proposition 3.1 and (4.6), the Bernstein estimates implied by $(\sqrt{\mathcal{B}})$ at primal and dual side, and the continuous/discontinuous gluing of basis functions over the interfaces between patches at primal and dual side, imply that for $s \in\left(-\min \left\{\frac{1}{2}, \tilde{\gamma}\right\}, \min \left\{\frac{3}{2}, \gamma\right\}\right)$ or $s \in\left(-\min \left\{\frac{3}{2}, \gamma\right\}, \min \left\{\frac{1}{2}, \tilde{\gamma}\right\}\right)$, the collections

$$
\bigcup_{j \geq-1} 2^{-s(j+1)} \Psi_{j}^{\Omega} \quad \text { or } \quad \bigcup_{j \geq-1} 2^{-s(j+1)} \tilde{\Psi}_{j}^{\Omega}
$$

are Riesz bases for $\mathcal{H}^{s}(\Omega)$ or $\tilde{\mathcal{H}}^{s}(\Omega)$, respectively (cf. DS98), assuming that, in the true manifold case, $s$ is such that this Sobolev space is defined.

The crucial property of the primal wavelets, as reflected in the title of this work, is given by the following result, which is a direct consequence of, for $j \geq 0$, $\mathcal{S}\left(\Psi_{j}^{\Omega}\right) \perp_{\langle\langle\cdot, \cdot\rangle} \mathcal{S}\left(\tilde{\Phi}_{j}^{\Omega}\right),(\underline{2.3})$, and $\tilde{\Phi}_{j}^{\Omega} \supset\left\{\tilde{\phi}_{j, x}^{\Omega}: x \in \bigcup_{q=1}^{M} \kappa_{q}\left(I_{j}^{\square} \cap \square\right)\right\}$.

Proposition 4.1. For all $j \in \mathbb{N}_{0}, x \in J_{j}^{\Omega}$ and $1 \leq q \leq M$,

$$
\left.\psi_{j, x}^{\Omega}\right|_{\Omega_{q}} \circ \kappa_{q} \perp_{L_{2}(\square)} Q_{\tilde{d}-1}(\square) .
$$

So the primal wavelets have the patchwise cancellation property of order $\tilde{d}$, which has the attractive consequences concerning matrix compression as discussed at the beginning of this paper.

\section{ILLUSTRATION}

We choose the simplest case from [DKU99] to illustrate the proposed construction, namely the case $d=\tilde{d}=2$ (and $r=1$; cf. (2.2)). Actually, we take the collections $\Phi_{j}, \tilde{\Phi}_{j}$ from DS99a, which differ from those from [DKU99] by basis transformations. These transformations have no influence on the new primal and dual scaling functions, also not after "gluing", and so also not on our wavelets, but they were essential for the construction of the composite wavelets in [DS99a, since they guarantee the analogue of $(\mathcal{V})$ at the dual side. The collection $\Phi_{j}$ spans the space of continuous piecewise linears with respect to an equidistant subdivision of the interval $[0,1]$ into $2^{j}$ intervals. With $I_{j}:=\left\{k 2^{-j}: k=0,1, \ldots, 2^{j}\right\}$, the primal 
scaling functions are defined by $\phi_{j, x}(y)=\delta_{x y} 2^{j / 2}\left(y \in I_{j}\right)$, i.e., they are the familiar "hat functions", except for the boundary function

$$
\phi_{j, 0}(x)=2^{j / 2} \begin{cases}1-2^{j} \cdot 7 x / 6, & 0 \leq x \leq 2^{-j} \\ 2^{j} \cdot x / 6-1 / 3, & 2^{-j}<x \leq 2 \cdot 2^{-j} \\ 0, & \text { elsewhere }\end{cases}
$$

and likewise $\phi_{j, 1}$. Applying a canonical ordering of the scaling functions, the primal and dual refinement relations are given by

$$
\begin{aligned}
& \left\langle\Phi_{j}, \tilde{\Phi}_{j+1}\right\rangle=\frac{1}{\sqrt{2}}\left[\begin{array}{ccccccc}
1 & 7 / 12 & -1 / 6 & -1 / 12 & & & \\
& 1 / 2 & 1 & 1 / 2 & & & \\
& & & 1 / 2 & 1 & 1 / 2 & \\
& & & & & & \ddots
\end{array}\right] \\
& \left\langle\tilde{\Phi}_{j}, \Phi_{j+1}\right\rangle=\frac{1}{\sqrt{2}}\left[\begin{array}{cccccccc}
1 & 3 / 2 & -3 / 4 & & & & & \\
& 1 / 2 & 3 / 2 & 1 / 2 & -1 / 4 & & & \\
& & -1 / 4 & 1 / 2 & 3 / 2 & 1 / 2 & -1 / 4 & \\
& & & & & & & \ddots
\end{array}\right] .
\end{aligned}
$$

To find suitably modified scaling functions, we can either apply Theorems 2.2 or 2.3 . that result in an update of $\phi_{j, 0}$ with a linear combination of 2 or 3 neighbouring scaling functions, respectively. We apply Theorem 2.3, which amounts to solving $\alpha_{j, 2^{-j}}$ and $\alpha_{j, 2 \cdot 2^{-j}}$ such that $\phi_{j, 0}+\alpha_{j, 2^{-j}} \phi_{j, 2^{-j}}+\alpha_{j, 2 \cdot 2^{-j}} \phi_{j, 2 \cdot 2^{-j}} \perp_{L_{2}(0,1)} P_{1}(0,1)$. From the discussion following the proof of Theorem 2.3 we know in advance that a unique solution exists. We found $\alpha_{j, 2^{-j}}=2 / 3$ and $\alpha_{j, 2 \cdot 2^{-j}}=-1 / 3$, so that the new boundary function is given by

$$
\phi_{j, 0}^{\text {new }}(x)=2^{j / 2} \begin{cases}1-2^{j} \cdot 11 x / 6, & 0 \leq x \leq 2^{-j} \\ 2^{j} \cdot 7 / 6 x-2, & 2^{-j}<x \leq 2 \cdot 2^{-j} \\ 1-2^{j} x / 3 & 2 \cdot 2^{-j}<x \leq 3 \cdot 2^{-j} \\ 0, & \text { elsewhere }\end{cases}
$$

and likewise $\phi_{j, 1}^{\text {new }}$. Making the corresponding transformation at the dual side indicated in (2.5) and (2.6), the new refinement matrices $\left\langle\Phi_{j}^{\text {new }}, \tilde{\Phi}_{j+1}^{\text {new }}\right\rangle,\left\langle\tilde{\Phi}_{j}^{\text {new }}, \Phi_{j+1}^{\text {new }}\right\rangle$ are given by

$$
\begin{aligned}
& \frac{1}{\sqrt{2}}\left[\begin{array}{ccccccc}
1 & 11 / 12 & -7 / 6 & -1 / 4 & 1 / 3 & 1 / 6 & \\
& 1 / 2 & 1 & 1 / 2 & & & \\
& & & 1 / 2 & 1 & 1 / 2 & \\
& & & & & & \ddots
\end{array}\right], \\
& \frac{1}{\sqrt{2}}\left[\begin{array}{cccccccccc}
-1 / 4 & 3 / 2 & -3 / 4 & & & & & & & \\
& 3 / 2 & 1 & 1 / 2 & -1 / 4 & & & & \\
& -1 / 2 & 0 & 1 / 2 & 3 / 2 & 1 / 2 & -1 / 4 & & & \\
& & & & -1 / 4 & 1 / 2 & 3 / 2 & 1 / 2 & -1 / 4 &
\end{array}\right]
\end{aligned}
$$

respectively. 

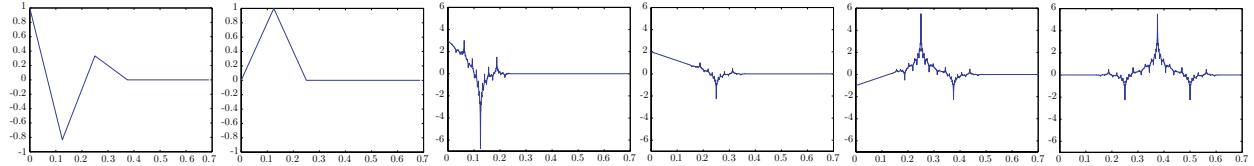

Figure 1. The first two primal (left) and the first four dual (right) new scaling functions.

The new scaling functions closest to the left boundary of $[0,1]$ are shown in Figure 1, where the second primal and the fourth dual scaling function correspond to the stationary functions on the line from CDF92. Note that there are three new dual scaling functions that do not vanish at 0 , whereas there is only one function in $\tilde{\Phi}_{j}$ with that property. The sets $\Phi_{j} \backslash\left\{\phi_{j, 0}^{\text {new }}, \phi_{j, 1}^{\text {new }}\right\}$ and $\tilde{\Phi}_{j} \backslash\left\{\tilde{\phi}_{j, 0}^{\text {new }}, \tilde{\phi}_{j, 1}^{\text {new }}\right\}$ are identical to the scaling functions constructed in DS98 to obtain wavelets with complementary boundary conditions (cf. Remark 2.4).

As a first application of the modified scaling functions to construct composite wavelets, we consider the one-dimensional domain $\Omega=[0,2]$ with patches $[0,1]$ and $[1,2]$, where we impose Dirichlet boundary conditions at $x=2$. The scaling functions are glued according to (4.4). Then, setting $J_{j}:=I_{j+1} \backslash I_{j}$, and using the canonical hierarchical basis as an initial complementary basis $\Xi_{j}$, we obtain the primal and dual wavelet functions by formulas (4.8), (4.9), and (4.7), taking into account the new refinement matrices $\left\langle\Phi_{j}^{\text {new }}, \tilde{\Phi}_{j+1}^{\text {new }}\right\rangle$ and $\left\langle\tilde{\Phi}_{j}^{\text {new }}, \Phi_{j+1}^{\text {new }}\right\rangle$ derived above. In the left picture of Figure 2, we illustrated the left boundary primal wavelet, one of both primal wavelets with support across the interface $x=1$ (the other one is obtained by reflection), and the right boundary primal wavelet satisfying the Dirichlet boundary condition, as well as their values at the "grid"-points defining the underlying partition. One may verify that indeed these wavelets have the patchwise cancellation property of order 2. In the right picture of Figure 2 one finds the corresponding dual wavelets. Note that the dual wavelet with support across the interface $x=1$ exhibits a jump.
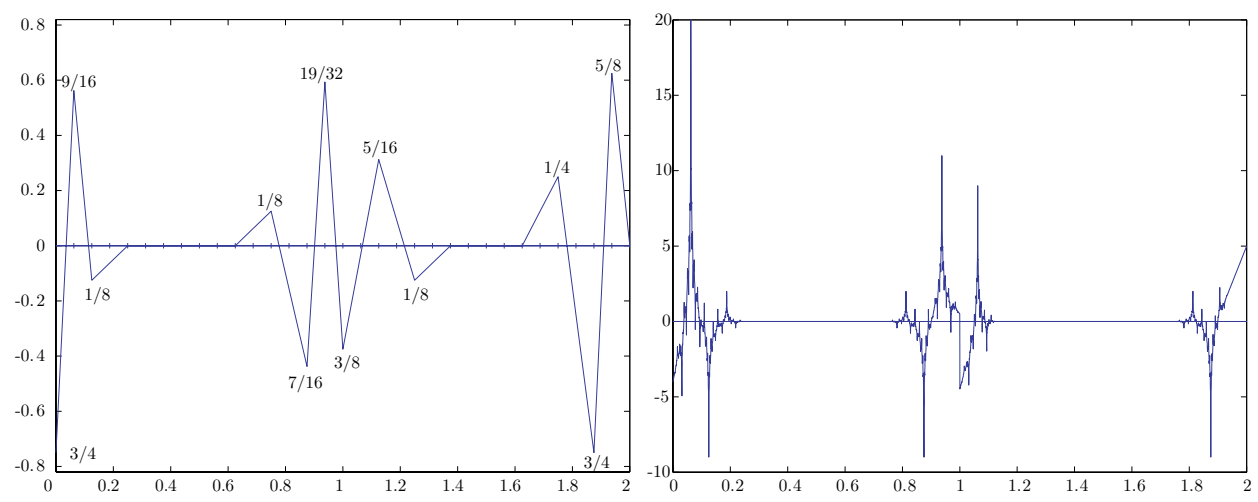

Figure 2. Primal (top) and corresponding dual (bottom) wavelets on $\Omega=[0,2]$ composed from the patches $[0,1]$ and $[1,2]$. 


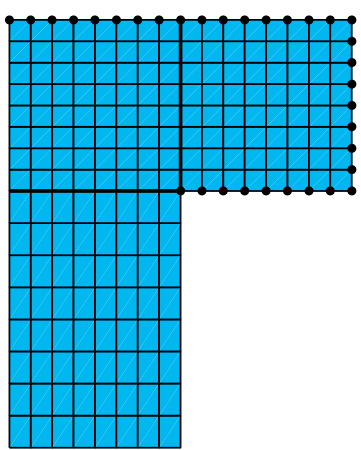

FiguRE 3. The L-shaped domain as a union of three patches.
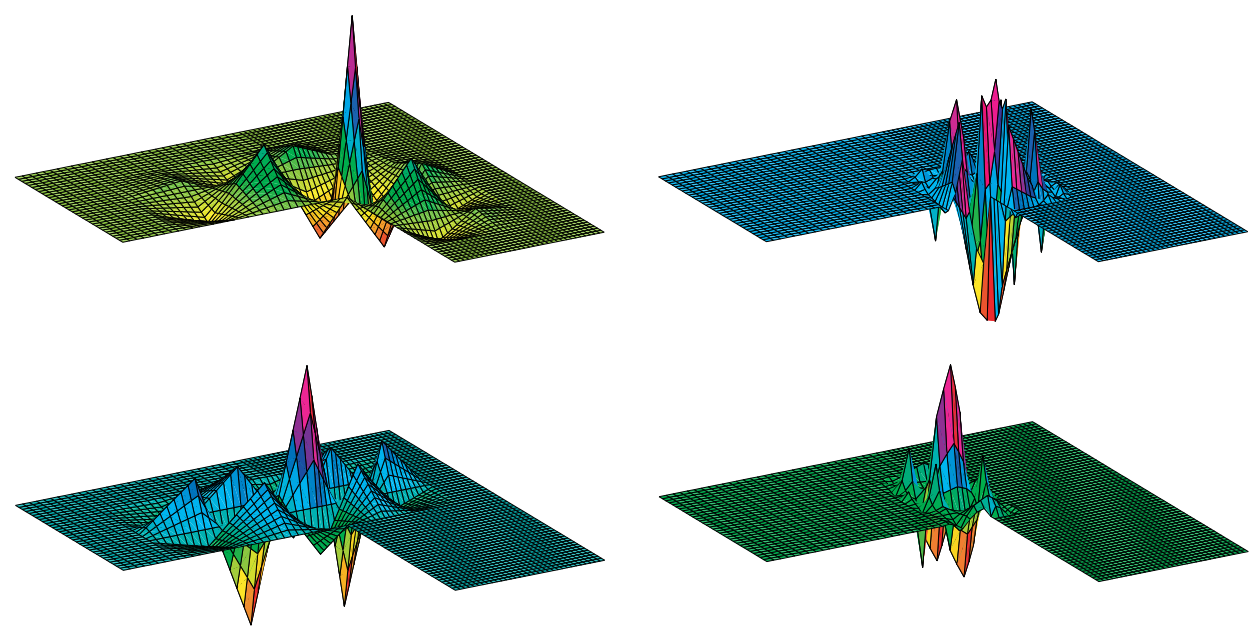

Figure 4. Primal (left) and corresponding dual (right) wavelets on the L-shaped domain near the re-entrant corner.

Next, we consider the two-dimensional L-shaped domain

$$
\Omega=([0,2] \times[0,2.5]) \backslash([1,2] \times[0,1.5]),
$$

which we represent by three patches according to Figure 3, On the faces indicated by the black bullets we prescribe Dirichlet boundary conditions. As set $\Xi_{j}^{\square}$ on the reference domain $\square=(0,1)^{2}$, we employ the standard choice $\left[\Phi_{j}^{\text {new }} \otimes \Xi_{j}, \Xi_{j} \otimes\right.$ $\Phi_{j}^{\text {new }}, \Xi_{j} \otimes \Xi_{j}$ ] (cf. Remark 3.2). Again, the primal and dual wavelets are given by the formulas (4.8), (4.9), and (4.7).

In Figure 4, we plotted two primal wavelets and their corresponding duals which live near the re-entrant corner $(x, y)=(1,1.5)$. Even though we have mixed boundary conditions in a neighbourhood of this vertex, these primal wavelets satisfy homogeneous Dirichlet boundary conditions everywhere. In Figure 5 we plotted two other primal wavelets, where one satisfies Dirichlet boundary conditions and the other does not. No dual wavelet satisfies Dirichlet boundary conditions.

The implementation of the wavelets follows the same line as that of the standard composite primal wavelets introduced in [DS99a]; see [Har01, HS04] for details. 


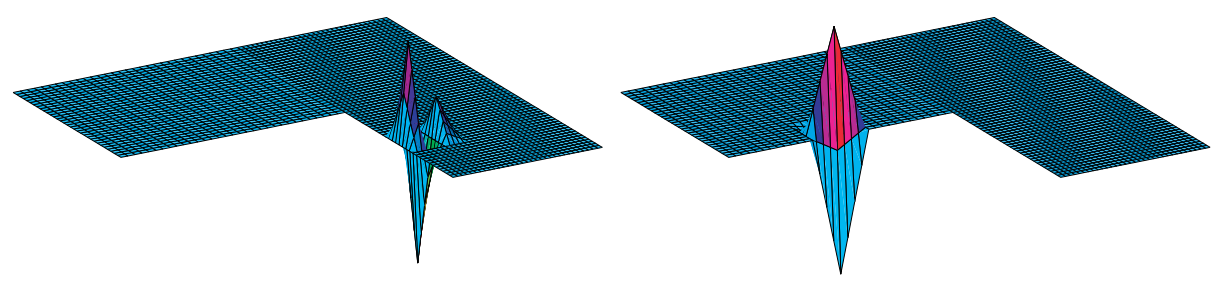

Figure 5. Wavelets with (left) and without (right) Dirichlet boundary conditions.

Note, however, that those standard composite primal wavelets that "meet" Dirichlet boundary conditions or, due to the jump in the Jacobians of the parametrizations, that have supports across the interface between the rectangle $[0,1] \times[0,1.5]$ and the square $[0,1] \times[1.5,2.5]$ have no cancellation properties with respect to the canonical $L_{2}(\Omega)$-scalar product. All our primal wavelets satisfy patchwise cancellation properties of order 2, and so in particular cancellation properties of order 2 with respect to the canonical $L_{2}(\Omega)$-scalar product. On the other hand, it is fair to say that, in this example, the $L_{2}(\Omega)$-condition number of our primal wavelets was about four times the $L_{2}(\Omega)$-condition number of the corresponding composite wavelets. For completeness, note that, due to the different dual spaces, the collection of wavelets on any given level constructed in this paper span a different space than the collection of standard composite wavelets on that level.

\section{REFERENCES}

[BF01] S. Bertoluzza and S. Falletta. Wavelets on $] 0,1[$ at large scales. Pubbl. 1212, I.A.N. Pavia, 2001.

[CDD01] A. Cohen, W. Dahmen, and R. DeVore. Adaptive wavelet methods for elliptic operator equations - Convergence rates. Math. Comp, 70:27-75, 2001. MR:1803124(2002h:65201)

[CDD02] A. Cohen, W. Dahmen, and R. DeVore. Adaptive wavelet methods II - Beyond the elliptic case. Found. Comput. Math., 2(3):203-245, 2002. MR1907380 (2003f:65212)

[CDF92] A. Cohen, I. Daubechies, and J.C. Feauveau. Biorthogonal bases of compactly supported wavelets. Comm. Pur. Appl. Math., 45:485-560, 1992. MR.1162365 (93e:42044)

[CDP96] J.M. Carnicer, W. Dahmen, and J.M. Peña. Local decomposition of refinable spaces and wavelets. Appl. and Comp. Harm. Anal., 3:127-153, 1996. MR1385049 (97f:42050)

[CM00] A. Cohen and R. Masson. Wavelet adaptive method for second order elliptic problems: Boundary conditions and domain decomposition. Numer. Math., 86:193-238, 2000. MR1777487 (2001j:65185)

[CTU99] C. Canuto, A. Tabacco, and K. Urban. The wavelet element method part I: Construction and analysis. Appl. Comput. Harmon. Anal., 6:1-52, 1999. MR.1664902 (99k:42055)

[DHS02] W. Dahmen, H. Harbrecht, and R. Schneider. Compression techniques for boundary integral equations - optimal complexity estimates. SIAM J. Numer. Anal., 43(6):22512271, 2006. MR2206435

[DKU99] W. Dahmen, A. Kunoth, and K. Urban. Biorthogonal spline-wavelets on the intervalstability and moment conditions. Appl. Comp. Harm. Anal., 6:132-196, 1999. MR:1676771 (99m:42046)

[DS98] W. Dahmen and R. Schneider. Wavelets with complementary boundary conditionsfunction spaces on the cube. Results Math., 34(3-4):255-293, 1998. MR.1652724 (99h:42057)

[DS99a] W. Dahmen and R. Schneider. Composite wavelet bases for operator equations. Math. Comp., 68:1533-1567, 1999. MR1648379 (99m:65122)

[DS99b] W. Dahmen and R. Schneider. Wavelets on manifolds I: Construction and domain decomposition. SIAM J. Math. Anal., 31:184-230, 1999. MR.1742299(2000k:65242) 
[GS04] T. Gantumur and R.P. Stevenson. Computation of differential operators in wavelet coordinates. Math. Comp., 75:697-709, 2006.

[GS05] T. Gantumur and R.P. Stevenson. Computation of singular integral operators in wavelet coordinates. Computing, 76:77-107, 2006. MR2174673

[Har01] H. Harbrecht. Wavelet Galerkin schemes for the boundary element method in three dimensions. Ph.D. Thesis, Technische Universität Chemnitz, Germany, 2001.

[HS04] H. Harbrecht and R. Schneider. Biorthogonal wavelet bases for the boundary element method, Math. Nachr. 269-270:167-188, 2004. MR2074780 (2005g:42080)

[KS04] A. Kunoth and J. Sahner. Wavelets on manifolds: An optimized construction. SFB 611 Preprint 163, Universität Bonn, July 2004. To appear in Math. Comp.

[Sch98] R. Schneider. Multiskalen- und Wavelet-Matrixkompression: Analysisbasierte Methoden zur Lösung großer vollbesetzter Gleigungssysteme. Habilitationsschrift, 1995. Advances in Numerical Mathematics. Teubner, Stuttgart, 1998.

[Ste04a] R.P. Stevenson. Composite wavelet bases with extended stability and cancellation properties. Technical Report 1304, Utrecht University, July 2004. To appear in in SIAM J. Numer. Anal.

[Ste04b] R.P. Stevenson. On the compressibility of operators in wavelet coordinates. SIAM J. Math. Anal., 35(5):1110-1132, 2004. MR2050194 (2005e:42128)

Institute of Computer Science and Applied Mathematics, Christian-Albrechts-UniVersity of Kiel, Olshausenstr. 40, 24098 Kiel, Germany

E-mail address: hh@numerik.uni-kiel.de

Department of Mathematics, Utrecht University, P.O. Box 80.010, NL-3508 TA Utrecht, The Netherlands

E-mail address: stevenson@math.uu.nl 